\title{
Trace Explosive Vapor Detection Using Silicon Nanowires in a Vertical Array with a Porous Electrode
}

\author{
Christopher R. Field ${ }^{1}$, Hyun Jin In ${ }^{2}$, Cy Tamanaha ${ }^{1}$, Susan L. Rose-Pehrsson ${ }^{1}$, Pehr E. Pehrsson ${ }^{1}$ \\ ${ }^{1}$ Naval Research Laboratory, Chemistry Division, Washington DC, 2037.5-5342 USA, \\ christopher.field@nrl.navy.mil \\ ${ }^{2}$ National Research Council, Post-Doctoral Research Associate, Washington, DC 20001
}

\begin{abstract}
:
The proliferation of improvised explosive devices (IEDs) and recent terrorist attempts like the infamous Shoe Bomber, Underwear Bomber, and Toner Cartridge Plot have demonstrated a significant need for portable and sensitive sensors for detection of explosive vapors. The Silicon Nanowires in a Vertical Array with a Porous Electrode (SiN-VAPOR) architecture has been developed to address this need with parts-per-billion detection limits for nitroaromatics, such as dinitrotoluene and trinitrotoluene (TNT). An initial slope method has been used to establish calibration curves for model compounds like nitrogen dioxide and ammonia and provides a mechanism for obtaining quantitative results with the SiN-VAPOR sensor.
\end{abstract}

Key words: Explosives, Sensor, Vapor, Nitroaromatics, Silicon Nanowires, Porous Electrode

\section{Introduction}

Field effect transistor-based chemical sensors (ChemFETs) based on nanowires have been researched for military, industrial, and commercial applications as gas phase chemical detectors. While nanowire-based ChemFETs have demonstrated an extreme sensitivity to vapors because of their large surface-to-volume ratios, they have continually underperformed as portable, field deployable sensors. The lack of performance is due to a combination of supporting substrate effects, humidity, $1 / f$ noise from a reduced number of charge carriers in single nanowire configurations, Schottky barrier formation at the contacts, and shot noise from wire-to-wire junctions in multiple, overlapping nanowire configurations.

The SiN-VAPOR sensor addresses these problems with a combination of Metal-Assisted Chemical Etched (MACE) nanowires and a twostage nanosphere lithography method [1,2]. Silicon nanowires are etched out of bulk silicon wafers in a vertical configuration. A porous top electrode is formed on top of the vertical nanowires creating a massive parallel array of nanowire sensors with rapid, uniform access to the analytes of interests. Fig. 1 shows a conceptual drawing of the architecture and a SEM image of the final sensor. The vertical configuration reduces substrate effects while maximizing sensing surface area. The massive parallel array eliminates noisy wire-to-wire junctions and increases the number of charge carriers.
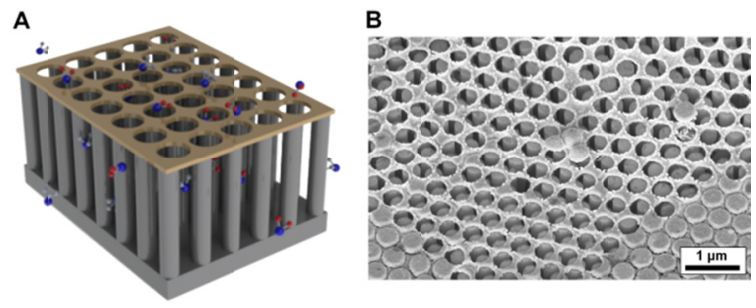

Fig 1. (A) A conceptual drawing of silicon nanowires in a vertical array with a porous top electrode. (B) $A$ scanning electron microscopy image of the porous top electrode in alignment with the metal-assisted chemical etched silicon nanowire array.

\section{Results}

A trace explosives test bed was developed to evaluate prototype sensor performance under a variety of environmental conditions. An impactor-inspired sample chamber with uniquely flexible electronics was used to evaluate the SiN-VAPOR sensor performance to nitrogen dioxide, ammonia, DNT, and TNT in humidified air. Fig. 2 shows the response to ammonia and nitrogen dioxide over the partsper-billion concentration range using the test bed and sample chamber. A GC-MS was modified to monitor analyte concentration in real-time during sensor evaluation. Fig. 3 shows the response of the SiN-VAPOR sensor to DNT in humidified air compared in real-time to the online GC-MS instrumentation. The SiN- 
VAPOR sensor responds similarly to the current commercially available instrumentation of explosive vapor detection.

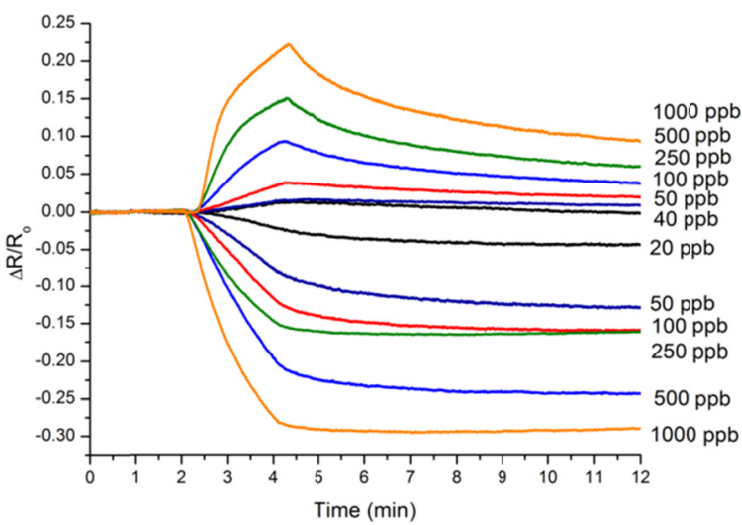

Fig. 2. Response to ammonia and nitrogen dioxide in humidified air at various concentrations in the partsper-billion concentration range. The sensor was exposed to 2 minutes of clean air followed by 2 minutes of analyte with a 10 minute recovery of clean air. The ammonia response is designated as an increase in resistance and the nitrogen dioxide exposure results in a decrease in resistance.

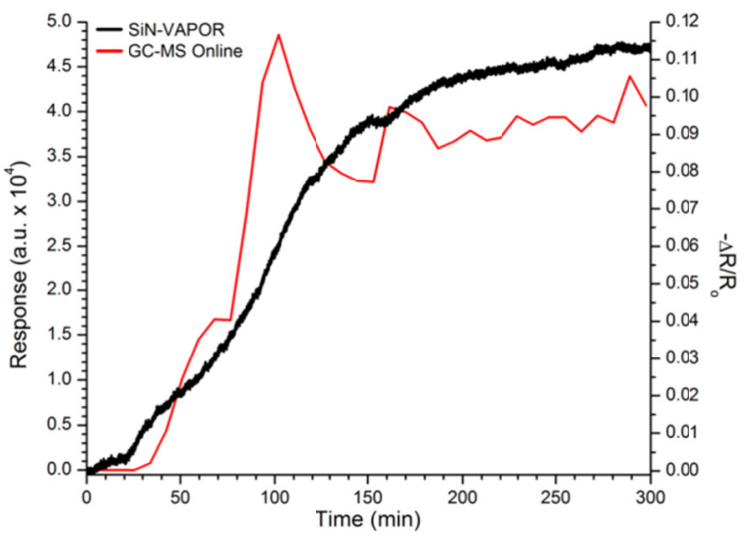

Fig. 3. The response to $40 \mathrm{ppb}$ of dinitrotoluene for the SiN-VAPOR sensor (black) and a modified GC$M S$ for online vapor detection (red) in humidified air.

\section{Acknowledgements}

The authors would like to acknowledge Michael Malito for his efforts in constructing the vapor delivery system. We would also like to thank Mark Hammond for his work on the computer interface connected to the vapor delivery system. This work was funded in part by the Defense Threat Reduction Agency. All fabrication was carried out in the Naval Research Laboratory's Nanoscience Institute.

\section{References}

[1] H.J. In, C.R. Field, P.E. Pehrsson, Nanotechnology 22, 355501-355507 (2011); doi:

[2] C.R. Field, H.J. In, N.J. Begue, P.E. Pehrsson, Analytical Chemistry 83, 4724-4728 (2011); doi: 10.1021/ac20079d 